\title{
INCOMPLETE INFORMATION GAMES WITH TRANSCENDENTAL VALUES*
}

\author{
JEAN-FRANÇOIS MERTENS AND SHMUEL ZAMIR
}

\section{CORE}

\begin{abstract}
In a repeated zero-sum two-person game with incomplete information on both sides, the asymptotic value is defined as $v=\lim _{n \rightarrow \infty} v_{n}$, where $v_{n}$ is the value of the game with $n$ repetitions. It is shown here that $v$ may be a transcendental number even for games in which all parameters defining the game are rational. This is in contrast to the situation in stochastic games where by the result of Bewley-Kohlberg [2] $v$ is algebraic. This indicates a fundamental difference between stochastic games and repeated games with incomplete information.
\end{abstract}

1. Introduction. Stochastic games are repeated games in which the payoff functions at each repetition may change according to the state in which the game is found. The current state of the game is known to all players and the transition probabilities between states from one repetition to the next one are determined by the moves of the players at that repetition (in a way known to all players).

Repeated games with incomplete information are also games in which the payoff functions depend on the state of nature which may be one of a given set of states. However, unlike in stochastic games, the state of nature, and hence the payoff function is unknown to the players but it is the same for all repetitions of the game. It is chosen at the very beginning of the game according to a prescribed probability distribution, and from that point on no transition to another state of nature takes place. The main issue in these games is the fact that each player is uncertain about the real state of nature, about which he has only partial information. This information is revised after each repetition as each player observes (directly or indirectly) the behaviour of the other players, that may reveal some of their knowledge about the real state of nature.

For repeated zero-sum two-person games $v_{n}$ denotes the value of the game consisting of $n$ repetitions in which the payoff function is defined as the average payoff per repetition. The asymptotic value of the game is defined as $v=\lim _{n \rightarrow \infty} v_{n}$, if this limit exists. The existence of $v$ for stochastic games with a finite number of states was proved in [2]. The existence of $v$ for various classes of repeated games with incomplete information was proved in [1], [3] and [4].

A special feature of the Bewley-Kohlberg proof [2] is that it is algebraic. In particular if the parameters of the game (i.e., payoffs and probabilities) are all rational numbers, then $v$ is a vector all of whose coordinates are algebraic numbers. Here we show that this is not true for repeated games with incomplete informationconstructing an actual example of a rational game with transcendental asymptotic value.

We are grateful to one of the referees for detecting an error in Figure 4.

2. The game. Consider the four $3 \times 4$ payoff matrices $\left\{A^{k l}\right\}, k=1,2, l=1,2$,

\footnotetext{
*Received November 13, 1979; revised May 6, 1980.

AMS 1980 subject classification. Primary 90D05.

IAOR 1973 subject classification. Main: Games.

OR/MS Index 1978 subject classification. Primary 236 Games/group decisions, noncooperative.

Key words. Repeated two-person zero-sum games, incomplete information, asymptotic values.
} 
where:

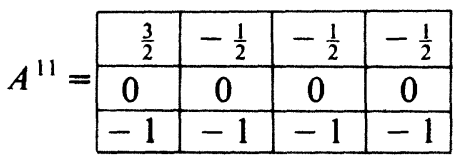

$A^{21}=$\begin{tabular}{|c|c|c|c|}
\hline$-\frac{1}{2}$ & $-\frac{1}{2}$ & $\frac{3}{2}$ & $-\frac{1}{2}$ \\
\hline-1 & -1 & -1 & -1 \\
\hline 0 & 0 & 0 & 0 \\
\hline
\end{tabular}

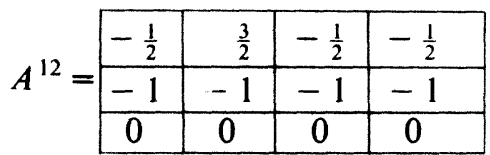

$A^{22}=$\begin{tabular}{|c|c|c|c|}
\hline$-\frac{1}{2}$ & $-\frac{1}{2}$ & $-\frac{1}{2}$ & $\frac{3}{2}$ \\
\hline 0 & 0 & 0 & 0 \\
\hline-1 & -1 & -1 & -1 \\
\hline
\end{tabular}

For each $(p, q), 0 \leqslant p \leqslant 1,0 \leqslant q \leqslant 1$ and for each positive integer $n$, consider the 2-person 0-sum game $\Gamma_{n}(p, q)$ defined as follows: At the beginning of the game, a chance move chooses a payoff matrix according to the probability distribution: $\operatorname{Pr}\left(A^{11}\right)=p q^{\prime}, \operatorname{Pr}\left(A^{12}\right)=p q, \operatorname{Pr}\left(A^{21}\right)=p^{\prime} q^{\prime}, \operatorname{Pr}\left(A^{22}\right)=p^{\prime} q$ (where throughout this paper we denote $p^{\prime}=1-p, q^{\prime}=1-q$ ). If the game $A^{k l}$ is chosen then player I (i.e., the maximizer and the row chooser) is informed what is the value of $k$ and player II is informed what is the value of $l$. Then the following procedure is repeated $n$ times: At stage $t(t=1,2, \ldots, n)$, player I chooses $i_{t} \in\{1,2,3\}$ and player II chooses $j_{t} \in$ $\{1,2,3,4\}$, then the pair $\left(i_{t}, j_{t}\right)$ is announced. After the $n$th stage player II pays player I $(1 / n) \sum_{t=1}^{n} a_{i j_{i}}^{k l}$, where $A^{k l}=\left(a_{i j}^{k l}\right)$ is the payoff matrix chosen by chance at the beginning of the game. Denote by $v_{n}(p, q)$ the value of $\Gamma_{n}(p, q)$.

A function $f$ defined on the unit square $S=\{(p, q) \mid 0 \leqslant p \leqslant 1,0 \leqslant q \leqslant 1\}$ is said to be concave in $p$ if for any $0 \leqslant q \leqslant 1, f(p, q)$ is a concave function on $0 \leqslant p \leqslant 1$. Similarly is defined the notion of convex function in $q$. For any $f$ defined on $S$ the concavification of $f$ (in $p$ ) is denoted by $\operatorname{Cav}_{p} f$ and is defined as the lowest function (pointwise) $\varphi$ which is concave in $p$ and satisfies $\varphi(p, q) \geqslant f(p, q) \forall(p, q) \in S$. Similarly $\operatorname{Vex}_{q} f$ denotes the convexification of $f$ (in $q$ ) which is the highest function $\psi$ which is convex (in $q$ ) and satisfies $\psi(p, q) \leqslant f(p, q), \forall(p, q) \in S$.

Coming back to our game, it belongs to the class of games treated in [4] from where we have the following result:

The asymptotic value $v(p, q)=\lim _{n \rightarrow \infty} v_{n}(p, q)$ exists for each $(p, q) \in S$ and is equal to the unique solution of the pair of functional equations:

(i) $v=\operatorname{Vex}_{q} \max (u, v)$

(ii) $v=\mathrm{Cav}_{p} \min (u, v)$,

where the function $u$ is the value of (ordinary) matrix game

$$
\Delta(p, q)=p q^{\prime} A^{11}+p q A^{12}+p^{\prime} q^{\prime} A^{21}+p^{\prime} q A^{22} .
$$

As usual, in the above statement $\max (u, v)$ and $\min (u, v)$ denote the pointwise maximum and minimum respectively of the functions $u$ and $v$.

REMARK. Note that $u(p, q)$ is the value of the game in which neither player is told anything about the choice of chance $A^{k l}$. Or, equivalently, the game in which the players are not allowed to use the information they have about the "real state of nature" $A^{k l}$.

For the game under consideration we have

$$
\Delta(p, q)=\begin{array}{|c|c|c|c|}
\hline 2 p q^{\prime}-\frac{1}{2} & 2 p q-\frac{1}{2} & 2 p^{\prime} q^{\prime}-\frac{1}{2} & 2 p^{\prime} q-\frac{1}{2} \\
\hline-p q-p^{\prime} q^{\prime} & -p q-p^{\prime} q^{\prime} & -p q-p^{\prime} q^{\prime} & -p q-p^{\prime} q^{\prime} \\
\hline-p q^{\prime}-p^{\prime} q & -p q^{\prime}-p^{\prime} q & -p q^{\prime}-p^{\prime} q^{\prime} & -p q^{\prime}-p^{\prime} q \\
\hline
\end{array}
$$

Note that $\Delta(p, q)$ is invariant under replacing $p$ by $p^{\prime}$ as well as under replacing $q$ by $q^{\prime}$. This implies that the value $u$ of $\Delta$ is a symmetric function both about $p=\frac{1}{2}$ and $q=\frac{1}{2}$. 


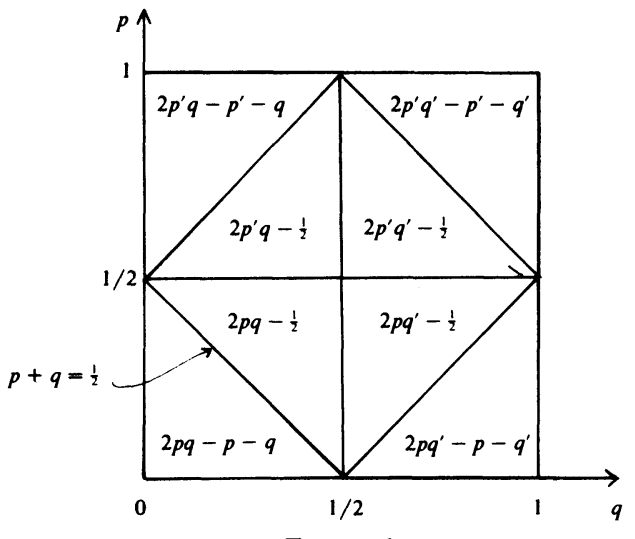

FiguRE 1

It is easily computed that the function $u$ is given as follows (see Figure 1):

$$
\begin{aligned}
& u(p, q)= \begin{cases}2 p q-\frac{1}{2} & \text { for } p+q \geqslant \frac{1}{2}, 0 \leqslant p \leqslant \frac{1}{2}, 0 \leqslant q \leqslant \frac{1}{2}, \\
2 p q-p-q & \text { for } p+q \leqslant \frac{1}{2}, 0 \leqslant p \leqslant \frac{1}{2}, 0 \leqslant q \leqslant \frac{1}{2},\end{cases} \\
& u(p, q)=u\left(p^{\prime} q\right)=u\left(p, q^{\prime}\right)=u\left(p^{\prime} q^{\prime}\right) .
\end{aligned}
$$

To compute $v=\lim _{n \rightarrow \infty} v_{n}$ we have now to solve the equations (i) and (ii). The technique for solving these equations is not quite a trivial matter and the interested reader can find it in the Appendix. The result is the function given in Figure 2.

Figure 2 is to be read as follows: The black thick lines are the locus of points $(p, q)$ on which $v(p, q)=u(p, q)$. This locus consists of the segments $p=0, p=1$, the curve $p=1 / 2[1-\ln (2-4 q)]$ on $\frac{1}{4} \leqslant q \leqslant \frac{1}{2}$ and its symmetries. At any point $(p, q)$ the value $v(p, q)$ is obtained by linear interpolation between the points of $v=u$, either in the $p$ direction or in the $q$ direction, as indicated by the arrows. The values in the various regions, computed according to the above mentioned convention, are also indicated in Figure 2. (For the regions on which $v$ is not specified, it is obtained by the symmetry $v(p, q)=v\left(p^{\prime}, q\right)=v\left(p, q^{\prime}\right)=v\left(p^{\prime}, q^{\prime}\right)$.)

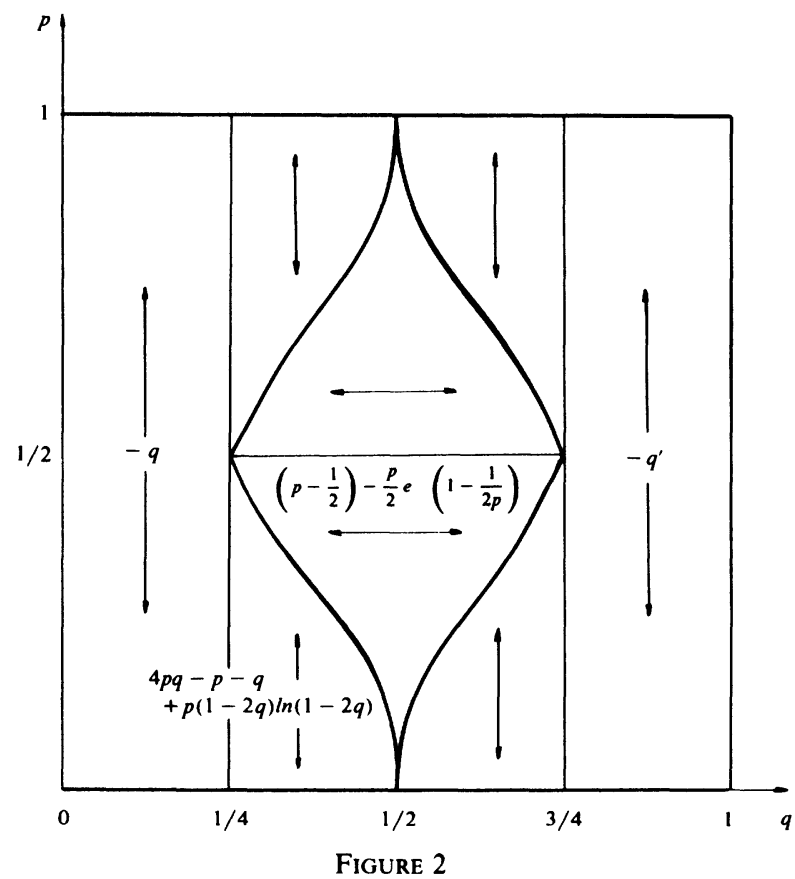


Note that although the computation of $v$ is not straightforward, once it is given, it is not too difficult to verify that it satisfies equations (i) and (ii) and, since these equations have a unique solution (see [4]), one has a relatively easy verification that this is in fact the asymptotic value.

We have thus obtained the desired result; for instance, at $p=\frac{1}{4}, q=\frac{3}{8}$, we have $v=-\frac{1}{4}-\ln 2 / 16$, and at $p=\frac{1}{4}, q=\frac{1}{2}$, we have $v=-\frac{1}{4}-1 / 8 e$, both transcendental numbers.

3. Remarks. (1) The example given here is of the "simplest" type of game possible. For incomplete information on one side (i.e., $p=0$ or $q=0$ ) one has by [1] that $\lim _{n \rightarrow \infty} v_{n}=\operatorname{Cav}_{p} u$ (or $\lim _{n \rightarrow \infty} v_{n}=\operatorname{Vex}_{q} u$ ). The operations Cav and Vex can be done algebraically and $u$ is a rational function in $p$ (or $q$ ), since it is the value of a matrix game whose entries are linear forms in $p$ (or $q$ ). Hence in this case the asymptotic value is necessarily an algebraic function.

Among the games with incomplete information on both sides our example falls both in what is known as the "independent case" and in the "standard information case" (see [4]). Mathematically, these two subcases are significantly simpler than the general case treated in [3].

(2) The Minmax and the Maxmin of the infinite games of the type treated here (see [1] and [5]) are, respectively, $\operatorname{Vex}_{q} \operatorname{Cav}_{p} u$ and $\operatorname{Cav}_{p} \operatorname{Vex}_{q} u$. As explained in the previous remark, these are always algebraic functions. In particular, it follows that if the infinite game has a value-i.e., if $\operatorname{Vex} \operatorname{Cav} u=\operatorname{Cav} \operatorname{Vex} u=v_{\infty}$-then $v_{\infty}$ is an algebraic function. Since in such a case we have also $v_{\infty}=\lim _{n \rightarrow \infty} v_{n}$ it follows that the phenomenon we have pointed out does not hold for this type of games when the infinite game has a value.

(3) In a wider class of repeated games with incomplete information, Sorin [7] has obtained games in which even the Minmax and the Maxmin values of the infinite game can be transcendental functions.

4. Appendix-The computation of $v(p, q)$. Before starting our computation of $v$, we remark again that we can find $v$ and prove that it is the required solution without proving all arguments that lead to the solution. It suffices to check that the suggested $v$ satisfies equations (i) and (ii), since these have a unique solution.

Let us now proceed to solve the equations:

(i) $v=\operatorname{Vex}_{q} \max (u, v)$,

(ii) $v=\mathrm{Cav}_{p} \min (u, v)$.

Proposition 1. $\operatorname{Cav}_{p} \operatorname{Vex}_{q} u \leqslant v \leqslant \operatorname{Vex}_{q} \mathrm{Cav}_{p} u$.

Proof. Note that both operators $\mathrm{Cav}_{p}$ and $\mathrm{Vex}_{q}$ are monotonic nondecreasing (i.e., $f \geqslant g \Rightarrow \operatorname{Cav} f \geqslant \operatorname{Cav} g$ and $\operatorname{Vex} f \geqslant \operatorname{Vex} g$ ). Thus by (i) we have: $v \geqslant \operatorname{Vex}_{q} u$. But by (ii) $v$ is concave in $p$, hence $v \geqslant \operatorname{Cav}_{p} \operatorname{Vex}_{q} u$. The other inequality follows in a similar way.

As we mentioned before $\operatorname{Cav} \operatorname{Vex} u$ and $\operatorname{Vex} \operatorname{Cav} u$ have a special significance; these are respectively the Maxmin and the Minmax of the infinite game.

We first compute those bounds. The computation is rather straightforward and the results are given in Figure 3 and Figure 4.

Since for $q \leqslant \frac{1}{4}$ and $q \geqslant \frac{3}{4}$ we found that $\operatorname{Vex} \operatorname{Cav} u=\operatorname{Cav} \operatorname{Vex} u$, it follows from Proposition 1 that $v(p, q)=-q$ for $q \leqslant \frac{1}{4}$ (and symmetrically for $q^{\prime} \leqslant \frac{1}{4}$ ). For the same reason, we have: $v=\operatorname{Vex} \operatorname{Cav} u=\operatorname{Cav} \operatorname{Vex} u=u$ on the lines $p=0$ and $p=1$.

We need now the following proposition:

Proposition 2. (a) On the regions on which $u<v, v$ is linear in the $p$ direction.

(b) On the regions on which $u>v$, $v$ is linear in the $q$ direction. 


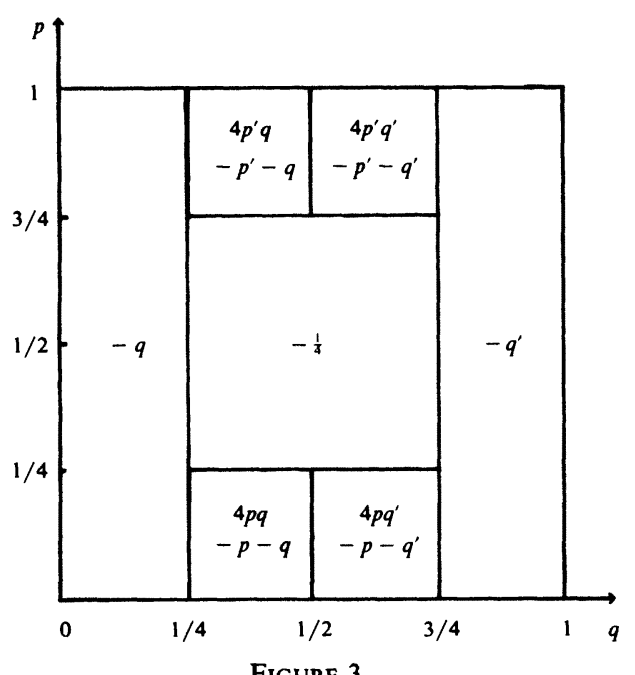

Figure 3

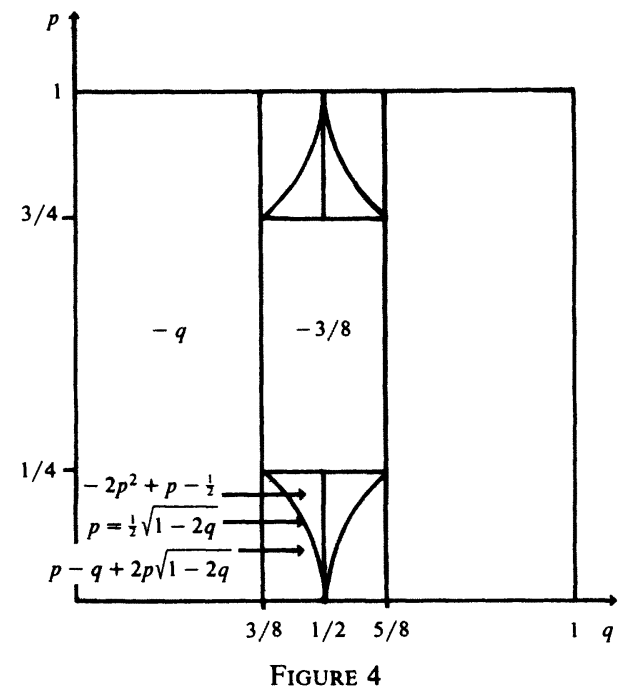

Figure 4

This proposition is quite apparent from the equations determining $v$. Formally it is a consequence of Lemma 6 in [4].

In view of Proposition 2, since both $u$ and $v$ are continuous, to compute $v$ it suffices to determine the locus of points $(p, q)$ on which $v(p, q)=u(p, q)$. The rest will be then determined by linear interpolations in the proper directions.

We now try to identify regions on the square according to whether $v>u, v<u$ or $v=u$ :

-On the lines $p=0$ and $p=1$ and at the points $\left(p=\frac{1}{2}, q=\frac{1}{4}\right)$ and $\left(p=\frac{1}{2}, q=\frac{3}{4}\right)$, we have $v=u$.

-On $\left\{(p, q) \mid p=\frac{1}{2}, \frac{1}{4}<q<\frac{3}{4}\right\}, u>\operatorname{Vex} \operatorname{Cav} u$. Hence, by Proposition $1, u>v$ on that region.

-On $\left\{(p, q) \mid q=\frac{1}{4}, 0<p<\frac{1}{2}\right\}, u<\operatorname{Cav}$ Vex $u$. Hence, by Proposition $1, u<v$ on that region. By the continuity of $u$ and $v$ we must have $u>v$ also on a neighborhood of the set for which $q>\frac{1}{4}$.

-Thus, there should be a line of $u=v$ which starts at $(p, q)=\left(\frac{1}{2}, \frac{1}{4}\right)$ and goes down rightwards (and, of course, there are the four symmetries of this line).

The general situation up to now seems to be as described in Figure 5.

Letting the line of $v=u$ in Figure 5 be $p=f(q)$ (in the first quarter; $p \leqslant \frac{1}{2}, q \leqslant \frac{1}{2}$ )

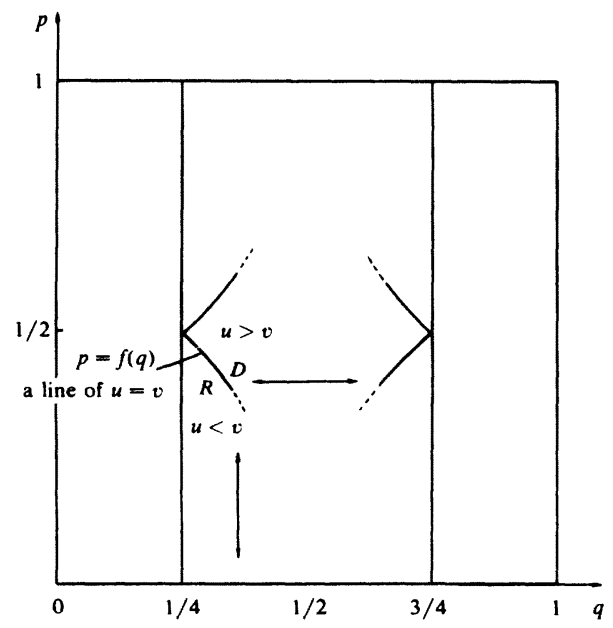

Figure 5 
and assuming that there is no such additional line below it except $p=0$, we can now write the function $v$ below and above the line $p=f(q)$ :

-Below $p=f(q)$ (the region $R$ in Figure 5), $u<v$, and thus $v$ is obtained by linear interpolation in the $p$ direction between $p=0$ and $p=f(q)$. Carrying out this interpolation yields:

$$
v(p, q)=2 p q-q-\frac{p}{2 f(q)}+\frac{p q}{f(q)} \quad \text { on } R
$$

-Above the line $p=f(q)$ (the region $D$ in Figure 5), $u>v$, and thus $v$ is obtained by linear interpolation in the $q$ direction between $p=f(q)$ and $p=f\left(q^{\prime}\right)$. But by symmetry $f\left(q^{\prime}\right)=f(q)$. Also by symmetry of $u$, we have that on the region $D, v(p, q)$ is constant in $q$. More explicitly,

$$
v(p, q)=u\left(p, f^{-1}(p)\right) \text { on } D .
$$

It remains now to find the function $f$. To do this we need the following:

Proposition 3. At any point $(p, q)$ at which $v(p, q)=u(p, q), v$ is differentiable if $u$ itself is differentiable provided that the line through $(p, q)$ on which $v=u$ is not parallel to one of the axes.

A proof of this last proposition can be found in [6]. In particular this proposition implies that $\partial v / \partial q$ is continuous across the line $p=f(q)$. By (1) and (2) this condition yields the differential equation:

$$
(1-2 q) f^{\prime}+4 f^{2}=0
$$

With the boundary condition $f\left(\frac{1}{4}\right)=\frac{1}{2}$, the solution is $f(q)=1 / 2[1-\ln (2-4 q)]$. Note that $\lim _{q \rightarrow 1 / 2} f(q)=0$. The function $v$ is now readily computed and verified to satisfy (i) and (ii).

\section{References}

[1] Aumann, R. J. and Maschler, M. (November 1968). Repeated Games with Incomplete InformationThe Zero-Sum Extensive Case. Report to the U.S. Arms Control and Disarmament Agency, Washington, D.C. Final report on contract ACDA/ST-143, prepared by Mathematica, Princeton, N.J., Chapter II, pp. 25-108.

[2] Bewley, T. and Kohlberg, E. (1976). The Asymptotic Theory of Stochastic Games. Math. Oper. Res. 1 197-208.

[3] Mertens, J. F. (1971/72). The Value of Two-Person Zero-Sum Repeated Games-The Extensive Case. Internat. J. Game Theory. 1 218-227.

[4] and Zamir, S. (1971). The Value of Two-Person Zero-Sum Games with Lack of Information on Both Sides. Internat. J. Game Theory. 1 39-64.

[5] - Minmax and Maxmin of Repeated Games with Incomplete Information. To appear in Internat. J. Game Theory.

[6] and Zamir, S. (to appear). Repeated Games.

[7] Sorin, S. Private communication.

CENTER FOR OPERATIONS RESEARCH AND ECONOMETRICS, CATHOLIC UNIVERSITY OF LOUVAIN, LOUVAIN-LA-NEUVE, BELGIUM 